\title{
Catalytically grown carbon nanotubes of small diameter have a high Young's modulus
}

Branimir Lukićl, Jin Won Seo ${ }^{1}$, Revathi R. Bacsa ${ }^{2}$, Sandrine Delpeux ${ }^{3}$, François Béguin ${ }^{3}$, Geoffroy Bister ${ }^{4}$, Antonio Fonseca ${ }^{4}$, Janos B. Nagy ${ }^{4}$, András Kis ${ }^{1}$, Sylvia Jeney ${ }^{1}$, Andrzej J. Kulik ${ }^{l}$, László Forróo ${ }^{1, *}$

${ }^{1}$ Institute of Physics of Complex Matter, Ecole Polytechnique Fédérale de Lausanne, 1015 Lausanne, Switzerland; ${ }^{2}$ Centre Interuniversitaire de Recherche et d'Ingénierie des Matériaux, Université Paul Sabatier, 31062 Toulouse Cedex 4, France; ${ }^{3}$ CRMD, CNRS-

Université, 1B rue de la Férollerie, 45071 Orléans Cedex 02, France; ${ }^{4}$ Facultés

Universitaires Notre-Dame de la Paix, 61 rue de Bruxelles, 5000 Namur, Belgium

* Corresponding author. E-mail: Laszlo.Forro@epfl.ch. Tel: ++41(21)6934306, Fax: $++41(21) 6934470$.

\section{Supporting Information.}

Summary of mechanical measurements. Diameter $D$, suspended length $L$, slope of the deflection-force curve $\Delta \delta / \Delta F$, calculated bending modulus $E_{B}$ and shear modulus $G$ for two batches of carbon nanotube bundles studied in this work.

\begin{tabular}{rrrrrr}
\hline \hline$D[\mathrm{~nm}]$ & $\begin{array}{r}L[\mathrm{~nm}] \\
\pm 10 \%\end{array}$ & $\begin{array}{r}\Delta \delta / \Delta F \\
{[\mathrm{~m} / \mathrm{N}]}\end{array}$ & $\begin{array}{r}E_{B}[\mathrm{GPa}] \\
\pm 50 \%\end{array}$ & $\begin{array}{r}G[\mathrm{GPa}] \\
\pm 50 \%\end{array}$ \\
\hline Batch I & 4.5 & 240 & 1.7 & 2060 & - \\
& 5.1 & 210 & 2.1 & 700 & - \\
7.2 & 190 & 7.7 & 35 & 0.17 \\
7.4 & 210 & 9.1 & 36 & 0.15 \\
7.6 & 250 & 5.9 & 85 & 0.29 \\
8.9 & 260 & 2.9 & 102 & 0.44 \\
9.8 & 285 & 3.8 & 69 & 0.29 \\
11.7 & 215 & 2.6 & 22 & 0.22 \\
12.9 & 280 & 3.1 & 27 & 0.20 \\
13.9 & 295 & 0.6 & 114 & 0.96 \\
15.0 & 300 & 0.8 & 68 & 0.61 \\
15.4 & 300 & 1.5 & 34 & 0.31 \\
16.1 & 285 & 2.0 & 18 & 0.20 \\
16.9 & 230 & 1.5 & 11 & 0.20 \\
17.2 & 250 & 1.2 & 15 & 0.24 \\
17.9 & 240 & 0.5 & 29 & 0.55
\end{tabular}




\begin{tabular}{lrrrrr} 
& 22.2 & 275 & 1.2 & 19 & 0.42 \\
\hline Batch II & 4.3 & 225 & 2.1 & 1624 & - \\
& 5.0 & 285 & 4.8 & 829 & - \\
& 7.4 & 225 & 3.2 & 123 & 0.51 \\
& 14.0 & 260 & 1.1 & 45 & 0.46 \\
& 25.4 & 245 & 0.8 & 7 & 0.23 \\
& 29.1 & 325 & 0.7 & 8 & 0.20 \\
\hline \hline
\end{tabular}

